\title{
ATRIBUIÇÃO DE EMOÇÕES A TRAÇOS FACIAIS ARTIFICIAIS
}

Luanna Marques MAGALHÃES, Laboratório de Psicologia Anomalística e Neurociências Universidade de Rio Verde, luanna_11_5@hotmail.com.

Lenny Francis Campos de ALVARENGA, Laboratório de Psicologia Anomalística e Neurociências, Universidade de Rio Verde.

Claudio Herbert NINA-E-SILVA, Laboratório de Psicologia Anomalística e Neurociências Universidade de Rio Verde.

\section{Recebido em: 30/10/2013 - Aprovado em: 20/12/2013 - Disponibilizado em: 15/01/2014}

Resumo: O antropomorfismo pode ser definido como sendo a atribuição de motivações, emoções e capacidades cognitivas consideradas tipicamente humanas a animais não-humanos. Todavia, os seres humanos também apresentariam a predisposição a atribuir características psicológicas humanas a objetos inanimados. Desse modo, o objetivo do presente estudo foi verificar a atribuição de emoções a traços faciais artificiais. Foram realizados dois experimentos. No Experimento 1, foi solicitado a 33 estudantes de graduação da Universidade de Rio Verde que atribuíssem emoções e/ou sentimentos a nove estímulos visuais diferentes compostos de traços faciais artificiais adaptados das faces de Chernoff. As respostas dos participantes foram categorizadas em "Positivo", "Neutro" e "Negativo". Os estímulos visuais representando traços faciais artificiais foram categorizados livremente pelos participantes na medida em que eram apresentados. Houve diferença significativa entre as médias de freqüência das categorias "Positivo" e "Negativo" $(F=13,56, p<0,05)$. Os traços faciais artificiais mais relevantes para a atribuição de sentimentos e emoções foram as simulações de "lábios". Já no Experimento 2, uma amostra de 28 estudantes de graduação da Universidade de Rio Verde foi solicitada a atribuir emoções a 11 estímulos visuais diferentes consistindo de traços faciais artificiais adaptados das faces de Chernoff. As respostas dos participantes foram categorizadas em "Positivo", "Neutro" e "Negativo". A maioria dos estímulos visuais representando traços faciais artificiais foi antropomorfizada pelos participantes. Não houve diferença significativa entre as médias de freqüência das categorias "Positivo" e "Negativo" ( $F=2,44, p>0,05)$. Assim como no Experimento 1, as simulações de "lábios" foram os traços faciais artificiais mais relevantes para a atribuição de emoções. Os presentes resultados estão de acordo com a literatura, segundo a qual as pessoas tenderiam a atribuir emoções humanas a objetos inanimados, tais quais os traços faciais artificiais utilizados no presente estudo.

Palavras-chave: antropomorfismo, cognição, emoções, Etologia Humana, faces de Chernoff.

Abstract: Anthropomorphism can be defined as assigning motivations, emotions and cognitive abilities considered typically human to non-human animals. However, humans would also show a predisposition to attribute human psychological characteristics to inanimate objects. Thus, the aim of this study was to determine the attribution of emotions to artificial facial features. Two experiments were conducted. In Experiment 1, we asked 33 undergraduate students to the University of Rio Verde to attribute emotions and / or feelings to nine different visual stimuli composed of artificial facial features adapted from Chernoff faces. The responses were categorized as " Positive ", " Neutral " and " Negative " The visual stimuli representing artificial facial features were categorized freely by the participants as they were presented. Significant difference between the mean frequency of the categories "Positive " and " Negative " ( F = $13.56, \mathrm{p}<.05)$. The artificial facial features most relevant to the attribution of feelings and emotions were simulations " lips ". In the second experiment, a sample of 28 undergraduate students at the University of Rio Verde was asked to assign emotions to 11 different visual stimuli consisting of artificial facial features adapted from Chernoff faces. The responses were categorized as " Positive ", " Neutral " and " Negative " . Most visual stimuli representing artificial facial features was anthropomorphized by the participants. There was no significant difference between the mean frequency of the categories " Positive " and "Negative " ( $F=2.44, p>.05)$. As in Experiment 1 , the simulations of " lips " were artificial facial features most relevant to the attribution of emotions. The present results are consistent with the literature, according to which people tend to attribute human emotions to inanimate objects such which artificial facial features used in this study.

Keywords: Anthropomorphism, cognition, emotions, Human Ethology, Chernoff faces.

\section{Introdução}

O antropomorfismo pode ser definido como sendo a atribuição de motivações, emoções e capacidades cognitivas consideradas tipicamente humanas a animais não-humanos (MENDES, ALVARENGA \& NINA-E-SILVA, 1999).

Tradicionalmente, as interpretações antropomórficas têm sido encaradas como sérios problemas metodológicos na Etologia e na Psicologia Animal Comparada (NINA-E- 
SILVA et al, 2000). Recentemente, contudo, as interpretações antropomórficas começaram a ser consideradas ferramentas de formulação de hipóteses e de interpretação comportamental tão válidas quanto a antroponegação (DE WAAL, 1997).

Os principais fatores predisponentes à categorização antropomórfica são a proximidade filogenética e o estereótipo cultural (MITCHELL \& HAMM, 1997). Enquanto a proximidade filogenética diz respeito ao fato de a antropomorfização ser mais freqüente para os comportamentos de animais mais filogeneticamente mais próximos do ser humano, o estereótipo cultural se relaciona às crenças que cada cultura possui a respeito de um determinado animal (MITCHELL \& HAMM, 1997).

Um estudo conduzido por Alvarenga et. al. (2000) entre os visitantes do Zoológico de Goiânia analisou esses dois fatores aplicados à realidade brasileira. Os resultados indicaram que houve mais relatos antropomórficos sobre o comportamento de mamíferos do que sobre os comportamentos de aves ou de répteis. Desse modo, as pessoas tenderam a antropomorfizar os comportamentos daqueles animais mais próximos de si mesmas filogeneticamente, como os macacos por exemplo (ALVARENGA et. al., 2000).

Por outro lado, os achados de Alvarenga et al. (2000) também corroboraram estudos estrangeiros anteriores sobre o estereótipo cultural de animais, visto que os carnívoros receberam comentários de juízos de valor mais depreciativos (e.g., "cruel", "malvado", "bravo") do que os herbívoros em exposição no Zoo de Goiânia.

Todavia, o antropomorfismo não se limita apenas à interpretação do comportamento de animais não-humanos. Também há antropomorfismo na percepção de objetos inanimados.

De acordo com Di Salvo e Gemperle (2003), os seres humanos tendem a perceber características psicológicas em certos objetos inanimados. As características físicas de um objeto, tais como forma e tamanho, influenciam na probabilidade de categorização antropomórfica por parte do percebedor (DI SALVO \& GEMPERLE, 2003).

O reconhecimento de emoções é uma característica que evoluiu no gênero humano para nos adaptar a diferentes contextos das relações interpessoais (NEUBERN, 2001). Na natureza, esta habilidade aumenta a probabilidade de sobrevivência e de perpetuação das espécies; mas para o gênero humano, uma das implicações desta habilidade é a adoção de julgamentos, comportamentos e atitudes que visam à análise de predisposições motivacionais do outro e nosso posicionamento em relação a estas.

Mesmo sendo de fundamental importância, esta habilidade não é estável ou estática em nossa espécie, pois alguns a apresentam em maior grau do que outros. Apesar disso, a precisão no reconhecimento 
de emoções é uma habilidade que pode ser treinada (EKMAN, 1991); mas para tanto é necessário estabelecer quais são as variáveis mais importantes para o reconhecimento facial de emoções.

A atribuição de sentimentos e emoções, dentre outras características psicológicas, também ocorre na percepção de objetos inanimados por conta de características e dimensões (DI SALVO \& GEMPERLE, 2003).

Dessa forma, o objetivo do presente estudo foi avaliar a atribuição de sentimentos e emoções a traços faciais artificiais. participaram da pesquisa 33 estudantes de graduação de ambos os gêneros e oriundos de vários cursos da Universidade de Rio Verde. A coleta de dados foi realizada em sala de aula da Universidade de Rio Verde que foi previamente preparada.

Os nove estímulos visuais apresentados aos participantes individualmente e que consistiram de traços faciais artificiais (vide Figura 1) foram elaborados no programa Microsoft PowerPoint 2007 a partir da adaptação das faces de Chernoff (1973).

\section{Materiais e Métodos}

Este estudo consistiu de dois experimentos. No Experimento 1,
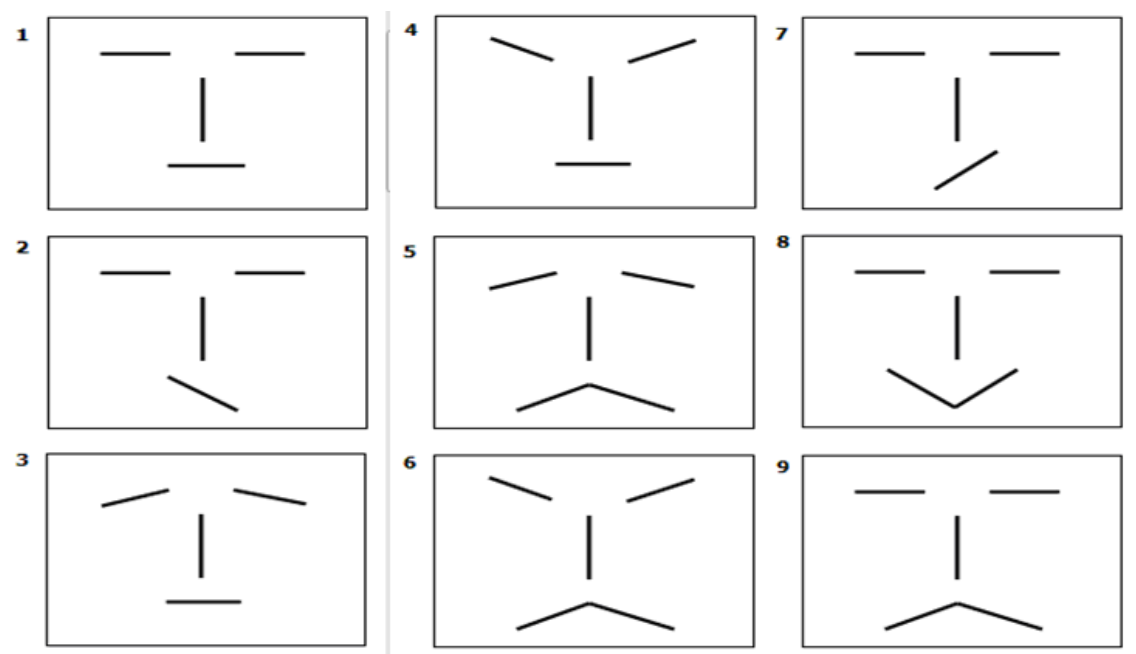

Figura 1. Estímulos visuais utilizados no Experimento 1 e elaborados a partir da adaptação das faces de Chernoff (1973).

Utilizou-se um computador portátil de tela LCD de 15,4" para apresentação dos estímulos visuais. Cada participante se sentou em uma carteira de sala de aula de frente para a tela do computador, enquanto um membro da equipe de pesquisa se sentou ao lado do computador e de frente para o participante, a fim de operar manualmente a apresentação dos estímulos visuais pelo computador portátil. 
Antes do início da apresentação, cada participante recebeu a seguinte instrução: "Será mostrado a você uma figura de cada vez e você deverá escrever que sentimento ou emoção cada uma das figuras demonstra. Não se preocupe, não há respostas certas ou erradas, portanto responda sem se preocupar". Caso o participante não tivesse compreendido a instrução, um membro da equipe de pesquisa procederia à releitura da instrução.

As respostas dos participantes foram agrupadas em três categorias distintas: positivo (emoções de bem-estar), negativo (emoções de mal-estar) e neutro (ausência de emoção ou sentimento).

Para fins de avaliação da freqüência de resposta aos estímulos visuais, considerou-se que as categorias "positivo" e "negativo" representavam a atribuição de emoções e
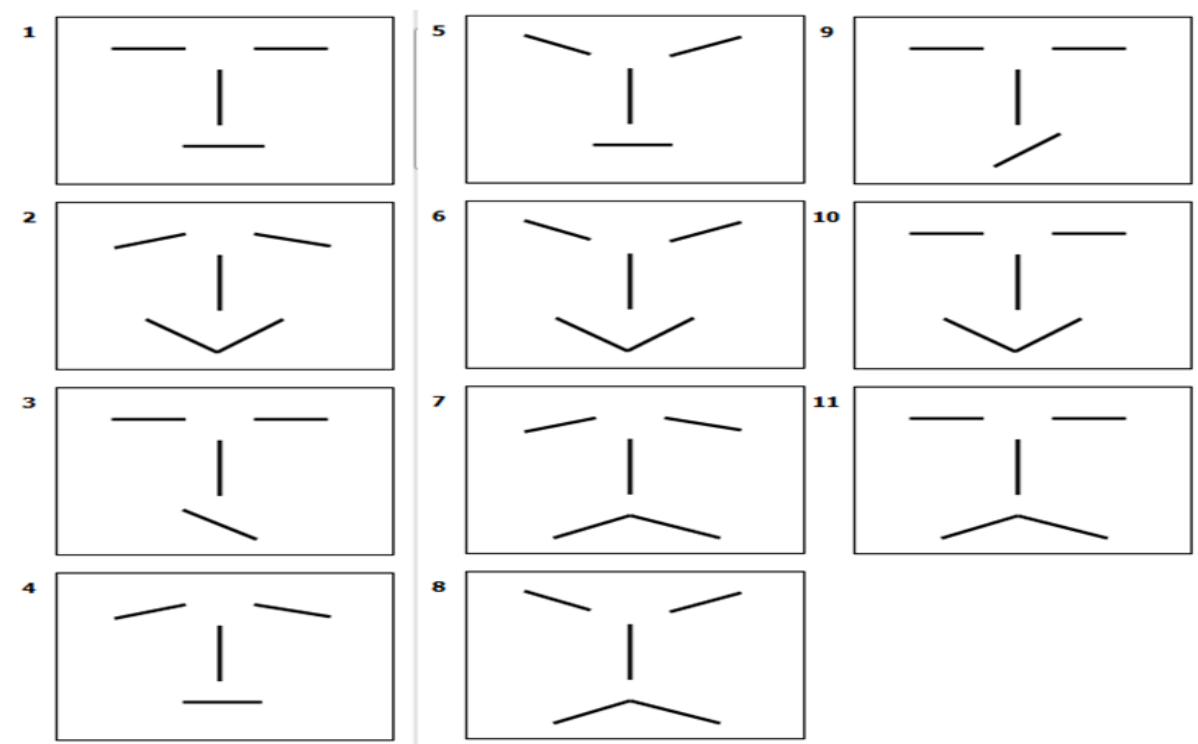

Figura 2. Estímulos visuais utilizados no Experimento 2 elaborados a partir da adaptação das faces de Chernoff (1973). 


\section{Resultados e Discussão}

\subsection{Experimento 1}

A Figura 3 demonstra que para a maioria dos estímulos visuais representando traços faciais artificiais foi atribuído sentimentos ou emoções classificadas como negativas (sendo $71 \%$ do total), e que apenas a figura 08 apresentou apenas atribuições positivas $(72 \%$ das classificações positivas) que foram controladas pela simulação do traço artificial "lábio". A análise de variância (ANOVA) indicou que houve diferença significativa entre as médias de freqüência das categorias "Positivo" e "Negativo" $(F=13,56, p<0,05)$.

A análise dos resultados da Figura 3 indicou que o traço facial artificial da figura 8 que controlou a atribuição de emoção foi a simulação de "lábio".

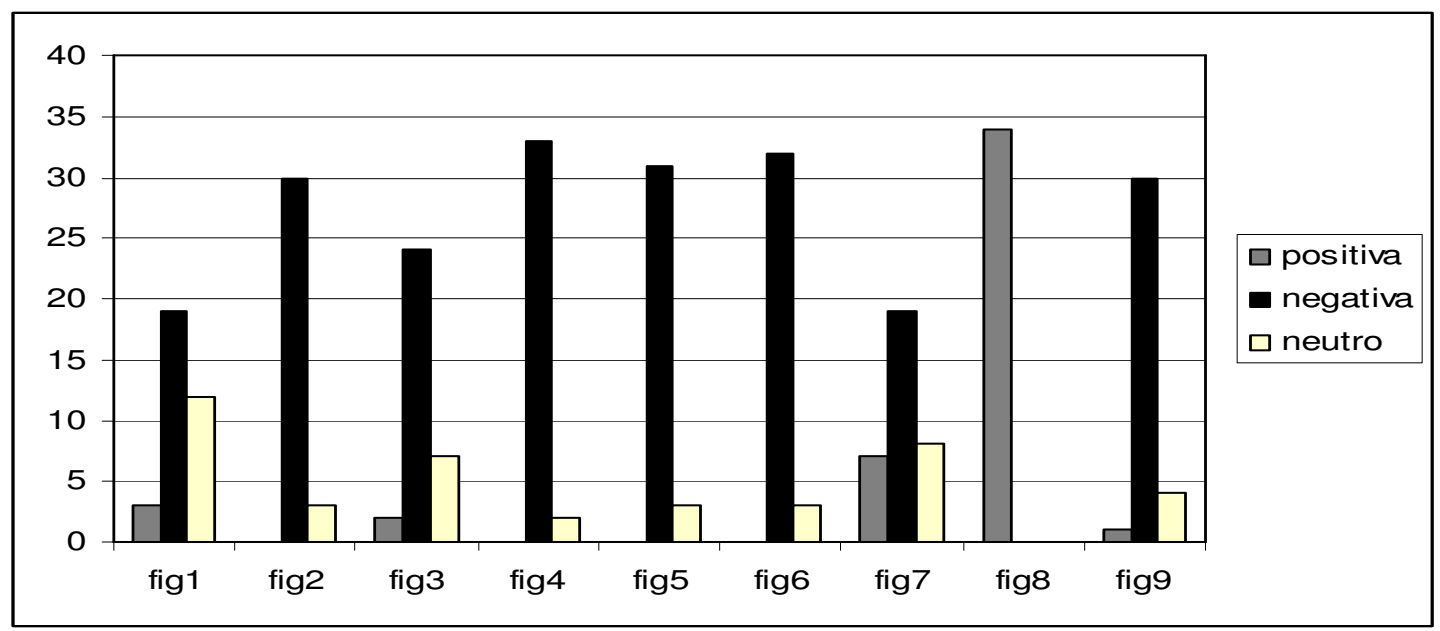

Figura 3. Freqüência de atribuição de sentimentos-emoções a cada um dos estímulos visuais representando traços faciais artificiais de acordo com as categorias "Positivo", "Neutro" e "Negativo" no Experimento 1.

Houve diferença significativa $(t=-1,97$; $p=0,05)$ entre as médias de atribuição de "raiva" e de "tristeza" a cada um dos estímulos visuais representando traços faciais.

A Figura 4 demonstra que a atribuição de raiva ocorreu majoritariamente apenas para as figuras 4 e 6 , demonstrando que o controle prioritário foi exercido pelo traço artificial dos "olhos-sobrancelhas", complementados, na figura 6, pelo traço artificial "lábio". 


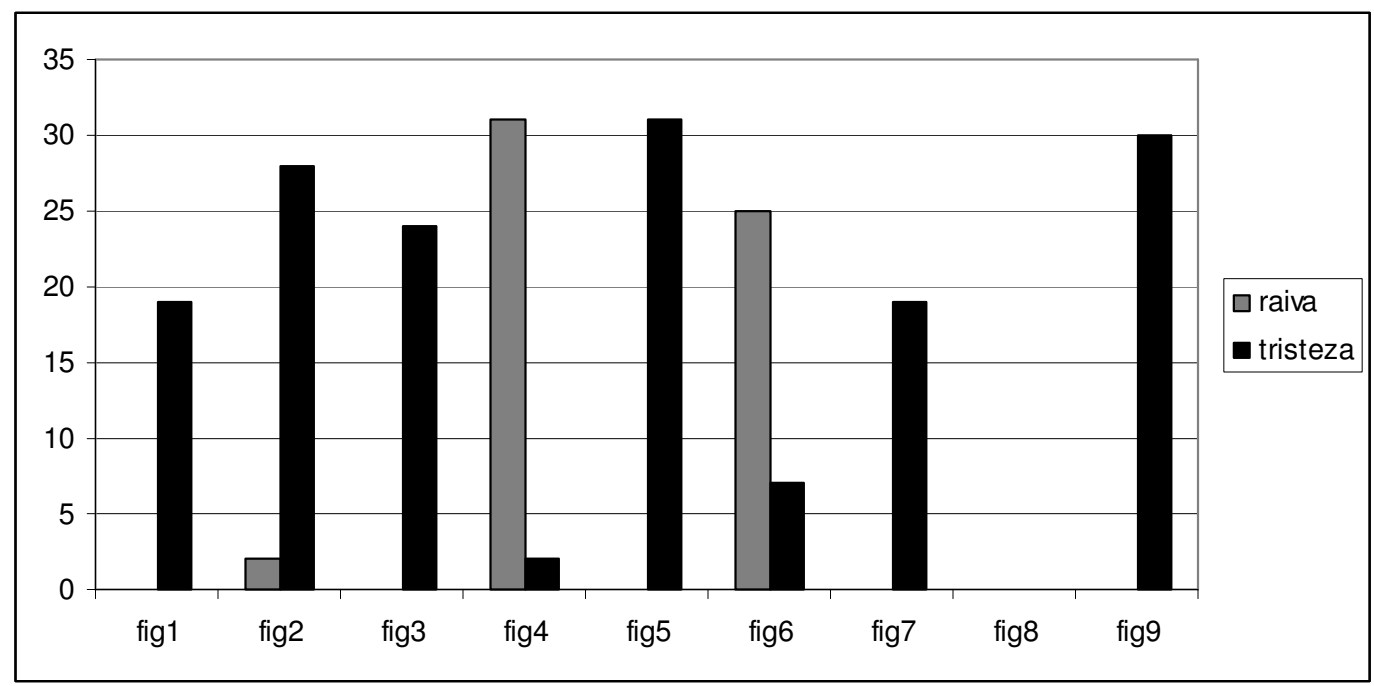

Figura 4. Freqüência de atribuição de sentimentos-emoções negativas (raiva e tristeza) a cada um dos estímulos visuais representando traços faciais no Experimento 1.

Os dados obtidos no Experimento 1 estão de acordo com a conclusão de Di Salvo e Gemperle (2003) de que humanos tendem a atribuir emoções e sentimentos a objetossímbolos não-humanos. Além disso, verificou-se que traços faciais artificiais baseados nos protótipos de faces de Chernoff (1973) se mostraram eficazes para a produção das atribuições de sentimentos e emoções.

\subsection{Experimento 2}

A maioria dos estímulos visuais representando traços faciais artificiais foi antropomorfizada pelos participantes.

A Figura 5 ilustra que apenas os estímulos visuais 1 e 9 receberam atribuição de emoção neutra por parte dos participantes. Contudo, a análise de variância (ANOVA) indicou que não houve diferença significativa entre as médias de freqüência das categorias "Positivo" e "Negativo" $(F=2,44, p>0,05)$.

A Tabela 1 ilustra os estímulos visuais considerados opostos e semelhantes em termos de percepção de emoção pelos participantes para um dos 11 estímulos visuais ilustrando traços faciais artificiais empregados no presente estudo. As dimensões "opostas" e "semelhantes" foram estabelecidas a partir do cálculo dos escores de correlação de Pearson entre cada estímulo visual e todos os demais.

A análise dos resultados da Tabela 1 indicou que os traços faciais artificiais mais relevantes para a atribuição de emoções foram as simulações de "lábios". 


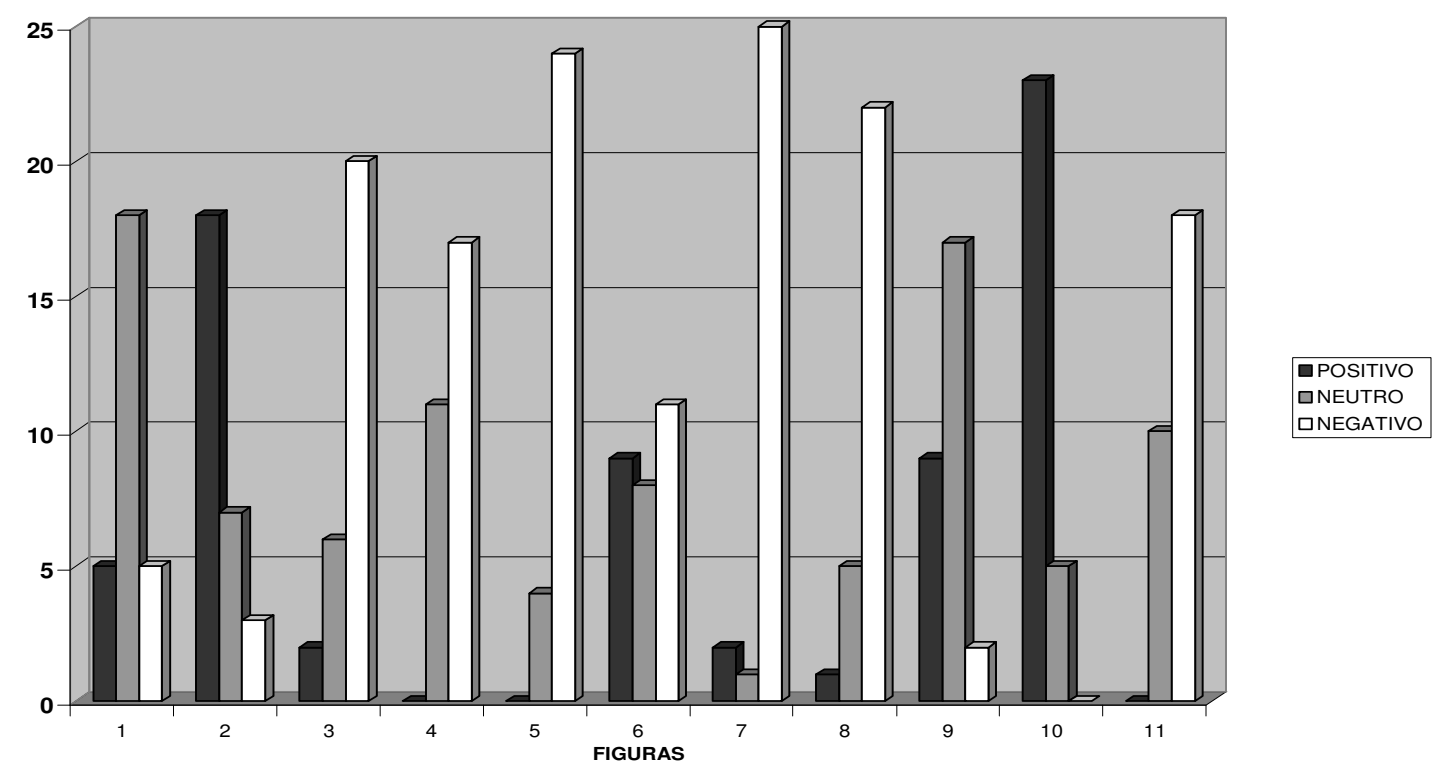

Figura 5. Frequiência de atribuição de emoções a cada um dos estímulos visuais representando traços faciais artificiais de acordo com as categorias "Positivo", "Neutro" e "Negativo" no Experimento 2.

De modo geral, os resultados do pessoas tenderiam a atribuir emoções Experimento 2 estão de acordo com Di Salvo humanas a objetos inanimados, tais quais os e Gemperle (2003), segundo os quais as traços faciais artificiais utilizados.

Tabela 1. Identificação das figuras opostas e semelhantes para cada um dos estímulos visuais apresentados aos participantes no Experimento 2 a partir dos escores de correlação de Pearson.

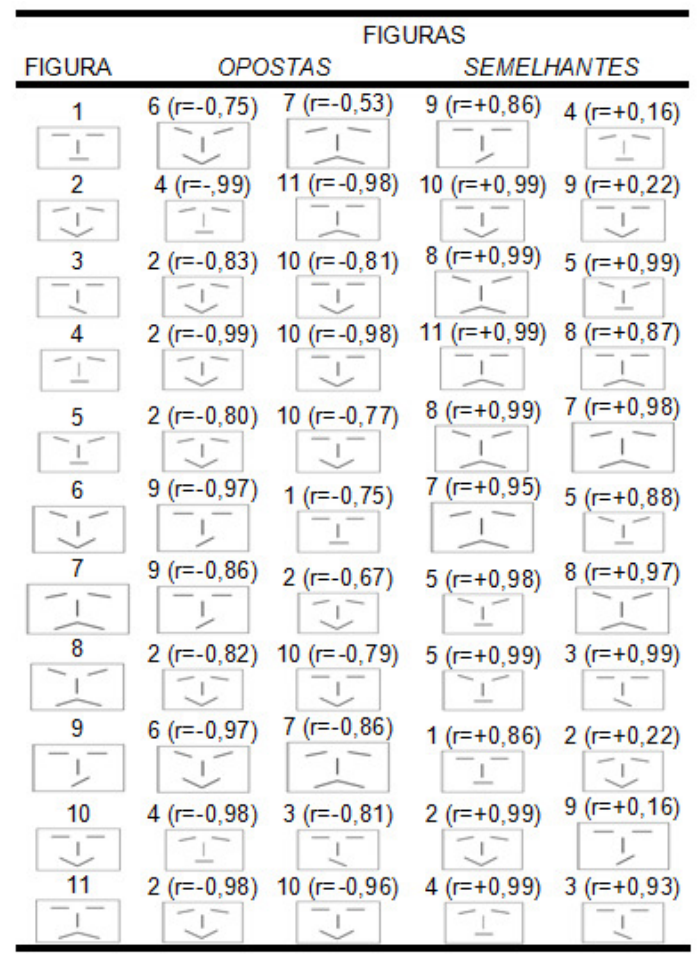


Da mesma forma que no Experimento 1, verificou-se que alguns aspectos prototípicos dos estímulos (tais como a simulação dos lábios) em detrimento de outros (tais como a simulação dos olhos) influenciaram mais na categorização antropomórfica pelos participantes (DI SALVO \& GEMPERLE, 2003).

\section{Conclusão}

Os resultados indicaram que os traços faciais artificiais adaptados das faces de Chernoff (1973) foram prioritariamente classificados como emoções ou sentimentos negativos. Sugere-se a realização de novos procedimentos experimentais que comparem traços faciais reais com os artificiais aqui utilizados para verificar que tipo de atribuições podem evocar.

Os resultados indicaram alto grau de antropomorfização dos traços faciais artificiais adaptados das faces de Chernoff (1973), o que possibilita a utilização desses estímulos visuais em novos estudos de simulação de antropomorfização.

\section{Referências Bibliográficas}

CHERNOFF, H. The use of faces to represent points in K-dimensional space graphically. Journal of the American Statistical Association, 68, 342, 361-378, 1973.

DISALVO, C.; GIAMPERLE, F. From seduction to fulfillment: the use of anthropomorphic form in design. DPPI, 23, 167-179, 2003.

EKMAN, P. \& O'SULLIVAN, M. Who can catch a lier? American Psychologist. 46, 9, 913-920, 1991.

MENDES; F.D.C.; ALVARENGA, L.F.C.; NINA-E-SILVA, C.H. Antropomorfismo em relatos d estagiários de etologia e análise experimental do comportamento. Anais de Etologia, 17, p. 132, 1999.

MITCHELL, R.W.; HAMM, M. The interpretation of animal psychology: anthropomorphism or behavior reading? Behaviour, 134, 173-174, 1997.

NEUBERN, M. S. O reconhecimento das emoções no cenário da psicologia: implicações epistemológicas e reflexões críticas. Psicologia, Ciência e profissão, v.21 n.2 Brasília jun. 2001

NINA-E-SILVA， C.H.; LOPES， D.M.; ALVARENGA， L.F.C.; NASCIMENTOJUNIOR, L.C.; MENDES, F.D.C. Categorização antropomórfica e diferenças de gênero. Anais de Etologia, 18, p. 89, 2000.

\begin{abstract}
ALVARENGA, L.F.C.; NINA-E-SILVA, C.H.; NASCIMENTO-JUNIOR, L.C.; VIEIRA, T.M. A influência da proximidade filogenética e do estereótipo cultural na interpretação antropomórfica de comportamentos animais reais. Anais de Etologia, 18, p. 212, 2000.
\end{abstract}

